\title{
Gene expression profiling in non-human primate jejunum, ileum and colon after total-body irradiation: a comparative study of segment-specific molecular and cellular responses
}

Junying Zheng ${ }^{1 *}$ D, Junru Wang ${ }^{1}$, Mylene Pouliot ${ }^{2}$, Simon Authier ${ }^{2}$, Daohong Zhou' ${ }^{1}$, David S. Loose ${ }^{3}$ and Martin Hauer-Jensen ${ }^{1,4}$

\begin{abstract}
Background: Although extensive studies have investigated radiation-induced injuries in particular gastrointestinal (GI) segments, a systematic comparison among the different segments on the basis of mode, magnitude and mechanism has not been reported. Here, a comparative study of segment-specific molecular and cellular responses was performed on jejunum, ileum and colon obtained at three time points (4, 7 and 12 days after irradiation) from non-human primate (Rhesus macaque) models exposed to 6.7 Gy or 7.4 Gy total body irradiation (TBI).

Results: Pathway analysis on the gene expression profiles identified radiation-induced time-, dose- and segmentdependent activation of tumor necrosis factor a (TNFa) cascade, tight junction, apoptosis, cell cycle control/DNA damage repair and coagulation system signaling. Activation of these signaling pathways suggests that colon sustained the severest mucosal barrier disruption and inflammation, and jejunum the greatest DNA damage, apoptosis and endothelial dysfunction. These more pronounced alterations correlate with the high incidence of macroscopic pathologies that are observed in the colon after TBI. Compared to colon and jejunum, ileum was resistant to radiation injury. In addition to the identification a marked increase of TNFa cascade, this study also identified radiation induced strikingly up-regulated tight junction gene CLDN2 (196-fold after 7.4-Gy TBI), matrix degradation genes such as MMP7 (increased 11- and 41-fold after 6.7-Gy and 7.4-Gy TBI), and anoikis mediated gene EDA2R that mediate mucosal shedding and barrier disruption.
\end{abstract}

Conclusions: This is the first systematic comparative study of the molecular and cellular responses to radiation injury in jejunum, ileum and colon. The strongest activation of TNFa cascades and the striking up-regulation of its down-stream matrix-dissociated genes suggest that TNFa modulation could be a target for mitigating radiation-induced mucosal barrier disruption.

Keywords: Total body irradiation, Tumor necrosis factor a, Gastrointestinal injury

\footnotetext{
* Correspondence: jzheng@uams.edu

'Division of Radiation Health, University of Arkansas for Medical Sciences, Little Rock, Arkansas 72205, USA

Full list of author information is available at the end of the article
} 


\section{Background}

Upon total-body irradiation (TBI), high-energy particles penetrate the body and directly or indirectly damage DNA and other key molecular structures within cells. Radiation causes death of rapidly proliferating cells and subsequent alterations that may lead to chronic disorders. The gastrointestinal (GI) system is among the most radiosensitive organs in the body $[1,2]$. Acute intestinal radiation injury is marked by massive cell death in the rapidly proliferating crypt epithelium and inflammation in the lamina propria [3-5]. The death of crypt cells results in insufficient replacement of the mucosal epithelium that leads to breakdown of the mucosal barrier and bacterial translocation $[6,7]$. In response to injury, the GI system initiates a series of compensated signaling cascades involved in cell death, inflammation, antimicrobial responses and tissue remodelling $[8,9]$. Activation of these signaling cascades is a spontaneous protective response of the host that could also exacerbate the destruction of tissues. For example, tumor necrosis factor $\alpha$ (TNF $\alpha$ ) promotes an immune response during infection, but excessive and unbalanced TNF $\alpha$ release can directly induce epithelial cell death and cause large-scale mucosal shedding, producing microerosions that cannot be compensated by epithelial tight junction rearrangement [10-13]. In addition, although apoptosis of massively DNA-damaged crypt cells prevents them from reentering the cell cycle [14], the toxins released from dead cells can cause damage to surrounding tissues, which could induce a second wave of cell death [15]. These events occur sequentially after radiation exposure and are governed by underlying cellular and molecular responses.

Because of the distinctive structures and functions of the GI tract segments, differences exist with respect to the cellular and molecular responses of each segment, depending on specific tissue radiosensitivity, damage mode, destruction magnitude and mechanism of injury. Therefore, the different GI segments undergo distinct types of damage after irradiation. Extensive studies have been conducted to investigate radiation-induced injuries in particular segments [5-8]. Small bowel is normally considered the most radiosensitive region due to its rapidly proliferating crypt cells $[1,5]$. It is also believed to be a major origin for gut-associated sepsis due to its lack of a thick protective mucous layer [16, 17]. Colon is thought to be more resistant to radiation injury, but symptoms associated with colon damage, such as diarrhea and hematochezia, have been suggested as indicators of survival after a radiation emergency [18]. To our knowledge, a systematic comparison among the different segments with respect to the mode, magnitude and mechanism of TBI-induced injury has not been reported. Understanding the dynamic activation of key signaling pathways and the cellular and molecular responses governing injury can aid in precisely predicting and evaluating the type, mode, magnitude and mechanism of injury, as well as in developing strategies needed to alleviate injuries along the GI tract. Furthermore, the above investigations support characterization of the pathophysiology of the acute radiation injury which is recognized as a regulatory requirement in drug development.

To help understand the mechanisms involved in acute intestinal radiation injury in the different GI tract segments as a basis for developing pharmacological modulators to mitigate the injury, we undertook a study in 24 non-human primate models in which the animals were exposed to $0 \mathrm{~Gy}, 6.7$ Gy or 7.4 Gy TBI. A total of 72 tRNA samples without pooling were collected from jejunum, ileum and colon at three time points (4, 7 and 12 days) after irradiation and subjected to gene expression profiling studies and comparative analysis of the gene expression profiles, including pathway analysis. Activation of signaling cascades suggests that colon undergoes the most severe mucosal barrier interruption and inflammation and that jejunum experiences the most DNA damage and repair, apoptosis and endothelial dysfunction. Compared to jejunum and colon, ileum is the most resistant intestinal segment to radiation injury.

\section{Results}

\section{Microarray data analysis}

The unirradiated jejunum, ileum and colon samples were used as the baseline for gene expression analysis. Genes with significantly changed mRNA levels were selected with a cut-off of $P<0.05$ and fold change $\geq 2.0$. The 18 lists of genes generated from the three segments on days 4, 7 and 12 after 6.7-Gy and 7.4-Gy TBI, including fold-change and P-values, are provided in Additional file 1: Table S1; Table 1 shows the 18 lists of genes that are in that file.

\section{Verification of gene expression by RT-qPCR}

Colon segments were selected for microarray validation. The expression of LCN15, EDA2R, HBB, HBA2, MMP12, GALC and ITM2A in control and samples irradiated at the different time points after 6.7-Gy and 7.4Gy TBI was validated by real-time RT-qPCR. In Fig. 1a, the expression of the selected transcripts at the different time points is plotted by normalized signals from the microarray (grey bars), together with their relative expression after normalization by GAPDH (black bars) in RT-qPCR. Figure $1 \mathrm{~b}$ demonstrates a statistics analysis to estimate the consistency between RT-qPCR results and the microarray data.

\section{Ingenuity pathway analysis (IPA) upstream regulator analysis}

IPA Upstream Regulator Analysis revealed that TBI induced extensive activation of TNF $\alpha$ cascades in colon at 
Table 1 Summary of 18 lists of genes altered $\geq 2.0-$ fold from jejunum, ileum and colon after exposure to 6.7-Gy and 7.4-Gy TBI. Available in Additional file 1

\begin{tabular}{llll}
\hline Time points & Jejunum & lleum & Colon \\
\hline 6.7 Gy_4d & 767 (478-up, 289-down) & 256 (141-up, 115-down) & 1306 (640-up, 666-down) \\
6.7 Gy_7d & 561 (206-up, 355-down) & 208 (71-up, 137-down) & 420 (196-up, 224-down) \\
6.7 Gy_12d & 409 (155-up, 254-down) & 622 (362-up, 260-down) & 454 (278-up, 176-down) \\
7.4 Gy_4d & 638 (456-up, 182-down) & 743 (468-up, 275-down) & 2574 (1263-up, 1311-down) \\
7.4 Gy_7d & 417 (162-up, 255-down) & 261 (71-up, 190-down) & 104 (47-up, 57-down) \\
7.4 Gy_12d & 488 (248-up, 240-down) & 520 (226-up, 294-down) & 539 (366-up, 173-down) \\
\hline
\end{tabular}

Note: The number outside the parenthesis is the total number of genes with mRNA level altered $\geq 2.0$-fold. Number in the parenthesis is the number genes with mRNA level up-regulated or down-regulated $\geq 2$.0-fold

day 4 (Fig. 2). Activation Z-score was calculated as a measure of functional and translational activation in Networks and Upstream Regulators Analysis. A Z-score of $<-2$ (inhibited) or $>2$ (activated) was considered as significant. Table 2 shows the Z-scores of each segment that predict TNF $\alpha$ activation in jejunum, ileum and colon after 6.7-Gy and 7.4-Gy TBI. TNF $\alpha$ cascades were strongly activated in all segments at day 4. Activation of TNF $\alpha$ was dose- and segment-dependent, with colon showing the strongest activation after 7.4-Gy TBI with a Z-score 9.3 and ileum the least activation after 6.7-Gy TBI with a Z-score 2.29. Activation of TNF $\alpha$ cascades were significantly suppressed on days 7 and 12 in all three gut regions (Table 2). In an unbiased analysis of activation of all of the upstream regulators, the top activated upstream regulators, in addition to $\mathrm{TNF} \alpha$, included interleukin $1 \mathrm{~B}$ and $1 \mathrm{~A}$ (IL1B and IL1A), with colon being the most activated and ileum the least activated segment (Fig. 3).

\section{IPA canonical pathway analysis \\ Activation of tight junction signaling in jejunum, ileum and colon}

As epithelial tight junctions play a key role in intestinal mucosal permeability $(25,26)$, we investigated the expression of tight junction-associated genes in the different GI segments after irradiation. The lists of transcripts altered $\geq 2$-fold at each time point were imported into IPA to identify the activation of tight junction signaling pathways. Analysis of the gene expression profiles revealed that tight junction signaling was only significantly activated in colon on day 4 after 7.4-Gy TBI; there was no significant activation in jejunum or ileum or at the other time points in colon (Fig. 4a). Figure 4b lists the altered genes corresponding to the tight junction pathway. Radiation induced very few altered genes in jejunum and ileum. However, 7.4Gy TBI induced 23 genes with mRNA level altered $\geq 2$-fold in colon at day 4 . Of these genes, CLDN2 was strikingly increased 105- and 196-fold after 6.7-Gy and 7.4-Gy TBI, respectively.
Expression of CLDN3, CLDN4, CLDN7 and CLDN8 were all significantly down-regulated. All of these changes were substantially attenuated on days 7 and 12 .

\section{Activation of immune response pathways in jejunum, ileum} and colon

The analysis by IPA of activation of immune response pathways in jejunum, ileum and colon showed that TBI induced a wide activation of these pathways in all three intestinal segments at day 4, with significant suppression of the activated pathways at days 7 and 12 (Fig. 5). The activation was dose- and region-dependent, with colon exposed to 7.4-Gy TBI showing the highest degree of pathway activation and ileum exposed to 6.7-Gy TBI showing the least activation. Various immune response pathways in jejunum were activated on day 4 after 6.7Gy TBI and were suppressed at day 7 (Fig. 5a). A comparative analysis of granulocyte adhesion and agranulocyte adhesion signaling in relation to diapedesis in jejunum, ileum and colon on days 4, 7 and 12 after 6.7-Gy and 7.4Gy TBI identified pathways significantly activated above the threshold -Log value ( $P$-value) (Fig. $5 \mathrm{~b}$, dashed line). Granulocyte and agranulocyte adhesion signaling and diapedesis were significantly activated at day 4 in all three segments, with colon the most activated site. These activations were significantly suppressed at days 7 and 12 . The altered genes regulating granulocyte adhesion and diapedesis pathways in jejunum, ileum and colon at days 4, 7 and 12 after 6.7-Gy and 7.4-Gy TBI are shown in Additional file 2: Table S2.

\section{Activation of aryl hydrocarbon receptor (AhR)-mediated apoptosis in jejunum, ileum and colon}

TBI induced significant activation of AhR-mediated apoptosis signaling pathway. AhR was strongly activated at day 4 after both TBI doses in all intestinal segments, with jejunum the most activated segment, and was significantly suppressed or attenuated in all segments at days 7 and 12 (Fig. 6a). Figure 6b lists the altered genes corresponding to the AhR signaling pathway. TBI also 


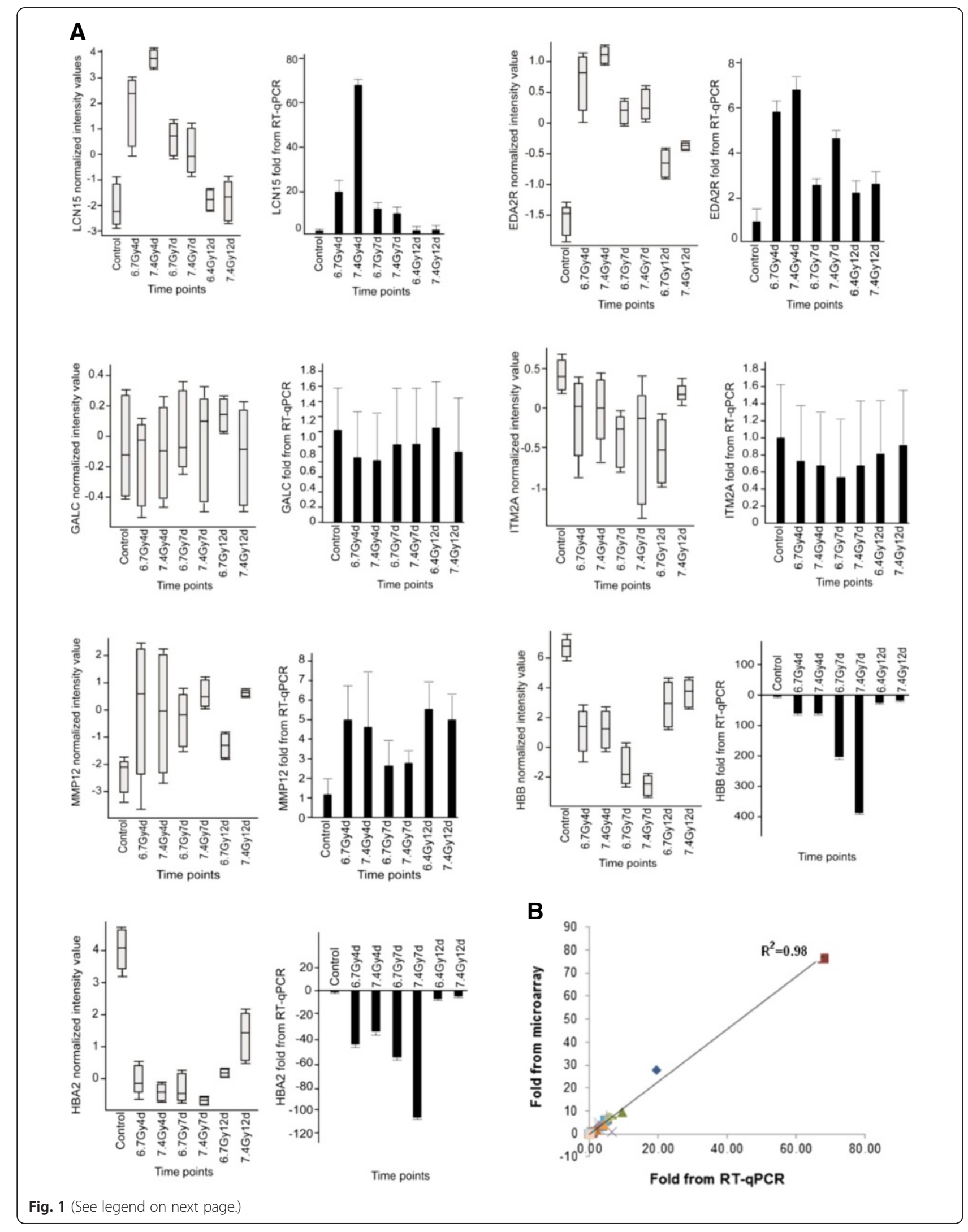


(See figure on previous page.)

Fig. 1 Validation of selected genes in colon by real-time RT-qPCR (a and $\mathbf{b}$ ). Expression of the selected genes at the different time points after TBI was plotted by microarray normalized signals (grey bars). The relative expression of the same selected genes was normalized by GAPDH gene at the different time points by real-time RT-qPCR (black bars). The opposite direction of HBB and HBA2 demonstrates their down-regulation after radiation (panel a). Statistics analysis was performed to estimate the consistency between RT-qPCR results and the microarray data (panel $\mathbf{b}$ )

activated other apoptotic signaling pathways, such as death receptor signaling (Fig. 6a); however, the magnitude of activation of these pathways was much lower than that of the AhR-mediated apoptosis pathway.

\section{Activation of cell cycle signaling pathways in jejunum, ileum and colon}

In response to radiation-induced DNA damage, pathways regulating cell cycle control and DNA damage repair signaling were activated. Activation of pathways regulating the G2/M DNA damage checkpoint regulation, mitotic roles of Polo-like kinases, and ATM signaling in jejunum was significant at day 7 at both TBI doses and was significantly suppressed at day 12 (Fig. 7). No significant activation of these pathways was observed in ileum or colon. Figure $7 \mathrm{~b}$ lists the genes whose expression were altered involved in the G2/M DNA damage checkpoint regulation pathway.

\section{Activation of coagulation system in jejunum, ileum and colon}

Analysis of cellular stress and injury signaling identified significant activation of the coagulation system in jejunum at day 4 after irradiation at both TBI doses, with complete suppression at days 7 and 12 (Fig. 8). Activation of the coagulation system was marginally significant in ileum or colon. Figure $8 \mathrm{~b}$ lists the genes whose expression were altered involved in the coagulation system.

\section{Up-regulation of anoikis signaling and TNF-mediated matrix-dissociated genes}

TBI induced significant up-regulation of EDA2R in all three segments after both radiation doses. EDA2R is a type III transmembrane protein of the TNF receptor superfamily that is associated with mediating anoikis, The increase of EDA2R mRNA expression reached a maximum at day 4 and gradually returned toward the baseline at days 7 and 12 (Fig. 9). Radiation also induced marked up-regulation of various matrix metalloproteinases (MMPs). The up-regulated MMPs in colon include MMP2, MMP7, MMP9 and MMP12 (Figs. 9 and Additional file 1: Table S1) and in jejunum MMP2 and MMP12. In ileum, only MMP2 was significantly induced. In addition to various types of MMPs, we identified a striking increase of LCN2 in colon at day 4 (58fold increase after 6.7-Gy TBI) (Fig. 2), which was significantly suppressed at day 12 . The striking increase of LCN2 only occurred in colon, not in jejunum and ileum
(Additional file 1: Table S1). IPA upstream analysis showed that these induced MMP genes and LCN2 were directly activated by TNF $\alpha$ (Fig. 2).

\section{Immunohistochemistry for TNFa}

Immunohistochemistry was performed on colon tissue sections from control and irradiated samples collected on study day 4 after 7.4-Gy TBI. Staining for TNFa was very weak across the normal colon section, except for some visible signals distributed as a streak underneath the intact mucosal epithelial lining (Fig. 10a, b). In the irradiated colon section, a significant increase in TNF $\alpha$ staining was observed in the mucosa, submucosa and serosa (Fig. 10c, d). In the mucosal region close to the epithelial barrier, TNF $\alpha$ had accumulated as a strong streak that formed an interface between swollen/disrupted cells and normal-shaped cells (Fig. 10c).

\section{Discussion}

This comparative analysis of the gene expression profiles of jejunum, ileum and colon obtained at three time points (4, 7 and 12 days) from Rhesus macaque models exposed to $6.7 \mathrm{~Gy}$ and 7.4 Gy TBI has yielded valuable insights into the differences in the radiosensitivity of these segments, as well as differences with respect to damage mode, destruction magnitude, and mechanism of injury. Pathway analysis of the gene expression profiles identified radiation-induced time-, dose- and segmentdependent activation of TNF $\alpha$ cascade, tight junction, apoptosis, cell cycle control/DNA damage repair and coagulation system signaling. On the basis of the activation of these signaling cascades and pathways, colon sustained the most severe mucosal barrier interruption and inflammation, and jejunum the most DNA damage, apoptosis and endothelial dysfunction. Compared to colon and jejunum, ileum was the most resistant segment to radiation exposure.

The TNF $\alpha$ cascade was identified as the upstream regulator of gene expression profile changes after TBI with the highest activation score as defined by IPA Upstream Regulator analysis. Activation of TNF $\alpha$ differed by intestinal segment, with colon showing the highest level of activation and ileum the lowest. TNF $\alpha$ cascade activation was also time-dependent, occurring at day 4 and then being completely suppressed at days 7 and 12 . TNF $\alpha$ is a pro-inflammatory molecule that regulates multiple cellular processes [9]. In intestine, it regulates inflammation, mucus secretion, cell survival and death 


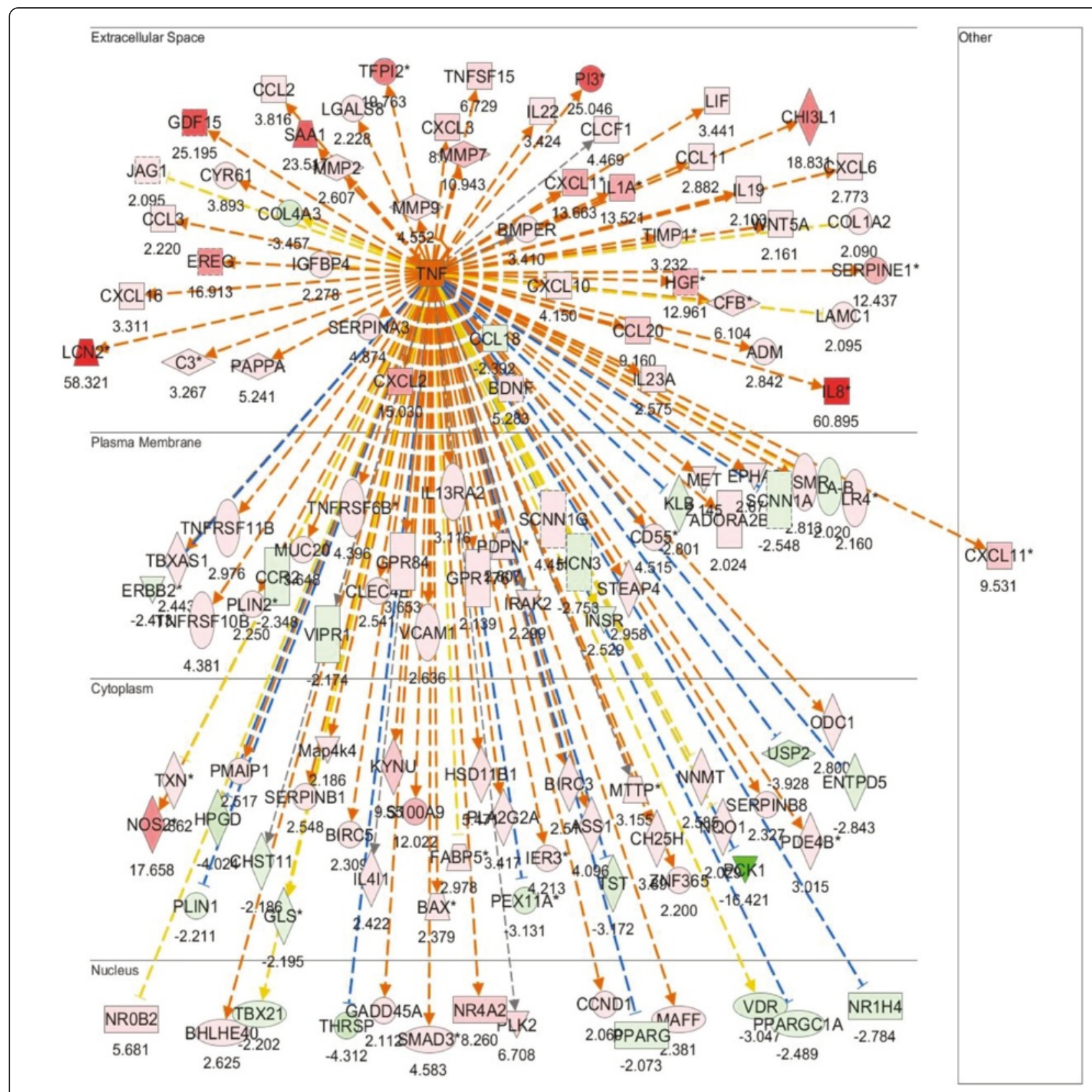

○ 2000-2014 Ingenuty Systems, inc. All rights reserved.

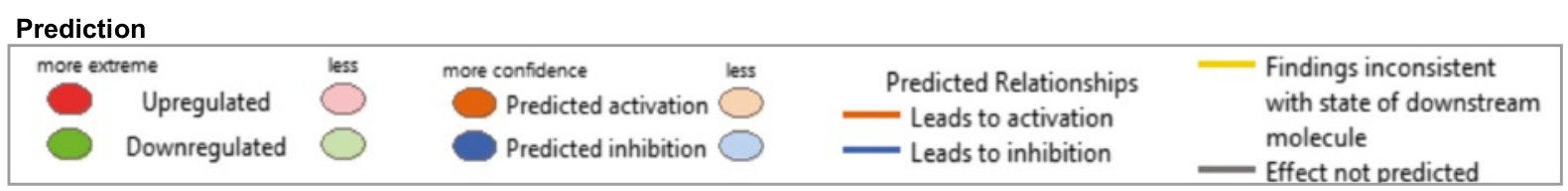

Fig. $2 \mathrm{TBI}$ at 6.7 Gy induced significant activation of TNFa cascades in colon tissue at day 4 (z-score, 6.7; P-value = 3.85e-23). An absolute z-score of $\geq 2$ is considered significant

[9-13]. Its protective or destructive role primarily depends on the dosage and activation of secondary signaling pathways. For example, TNFa overexpression by exogenous administration or specific knock-in induces abnormal epithelial shedding producing microerosions that cannot be sealed by tight junction rearrangement [19]. Clinically, many studies have implicated TNFo's intricate link to intestinal barrier disruption in inflammatory 
Table 2 Dynamic activation Z-scores ${ }^{a}$ of TNFa cascades in jejunum, ileum and colon at days 4, 7, and 12 after 6.7 Gy and 7.4 Gy TBI

\begin{tabular}{|c|c|c|c|c|c|c|}
\hline \multirow{2}{*}{$\begin{array}{l}\text { Time } \\
\text { points } \\
\text { (d) } \\
\end{array}$} & \multicolumn{2}{|c|}{ Z-score (Jejunum) } & \multicolumn{2}{|c|}{ Z-score (lleum) } & \multicolumn{2}{|c|}{ Z-score (Colon) } \\
\hline & $6.7 \mathrm{~Gy}$ & 7.4 Gy & $6.7 \mathrm{~Gy}$ & $7.4 \mathrm{~Gy}$ & $6.7 \mathrm{~Gy}$ & $7.4 \mathrm{~Gy}$ \\
\hline 4 & 6.46 & 5.32 & 2.29 & 3.8 & 7.9 & 9.3 \\
\hline 7 & 1.26 & -0.89 & -0.31 & -1.06 & 1.9 & 1.39 \\
\hline 12 & -0.88 & 0.29 & 0.38 & 0.89 & 1.26 & 0.45 \\
\hline
\end{tabular}

${ }^{a}$ An absolute Z-score of $\geq 2$ is considered significant. An upstream regulator is predicted to be activated if the $Z$-score is $\geq 2$ and inhibited if the $Z$-score $\leq-2$

bowel diseases such as Crohn's disease and ulcerative colitis [9]. In irradiated colon, where the TNF $\alpha$ cascade was highly activated, histology revealed large-scale epithelial shedding with more severe barrier disruption than in other GI segments.

The distribution pattern of immunohistochemical staining for TNF $\alpha$ in irradiated colon demonstrates a direct involvement of TNF $\alpha$ in radiation-induced mucosal shedding. At day 4 after 7.4 Gy TBI, the interstitial space from serosa to mucosa showed a marked increase of TNF $\alpha$ staining, and, in an intriguing finding, a strong streak representing accumulation of TNF $\alpha$ was observed in the mucosal region close to the epithelial barrier, separating abnormally from normally shaped cells (see Fig. 10). Regulation of intestinal inflammation and mucosal injury by TNF $\alpha$ has undergone extensive study. Targeting of TNF $\alpha$ with antibodies has been successfully used in the clinical treatment of inflammatory bowel disease for many years, highlighting the importance and clinical relevance of TNFo's function in intestinal inflammation [20]. Our finding in normal colon of a visible streak of TNFa closely adjacent to the base of the epithelial lining (see Fig. 10a, b) suggests that TNFo could act as a sentinel in the response to barrier disruption.

Of the TNF $\alpha$ downstream targets, genes regulating cellular dissociation including MMP2, MMP3, MMP7, MMP9 and LCN2 were strikingly increased in colon tissue after 7.4 Gy irradiation. Among these in colon, MMP7 was increased 11- and 41-fold after 6.7 and 7.4 Gy TBI, and LCN2 was increased 58- and 52-fold, respectively. Epithelial MMP-7 breaks down extracellular matrix (ECM) and activates $\alpha$-defensin produced by paneth cells [21]. LCN2 protein, also called NGAL (neutrophil gelatinase-associated lipocalin), can form a complex with MMP-9 and strongly enhance and preserve MMP-9 activity [22]. The strikingly increased expression

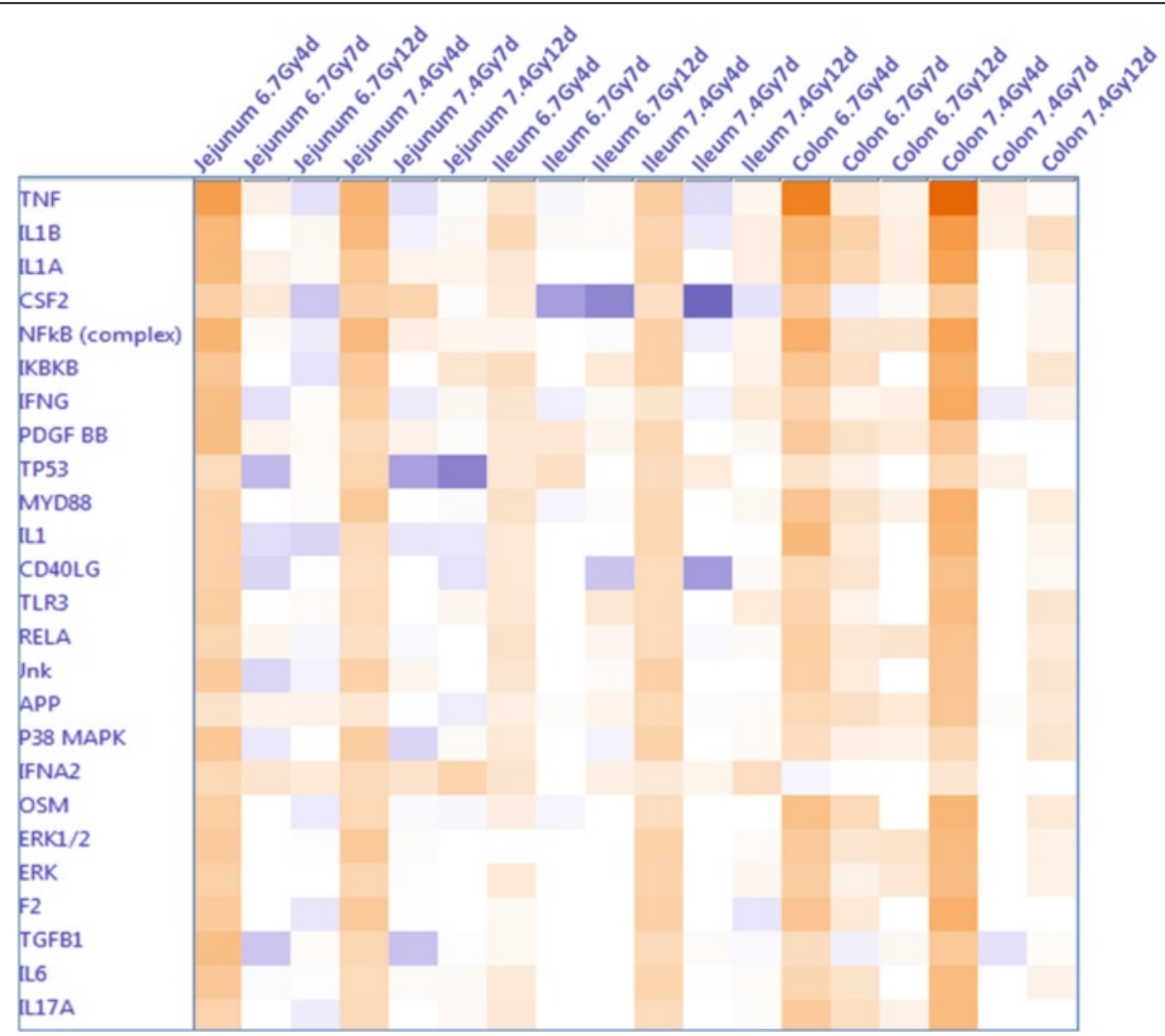

Fig. 3 Comparative analysis of the dynamic activation of upstream regulators in jejunum, ileum and colon after 6.7 Gy and 7.4 Gy TBI. An absolute $z$-score of $\geq 2$ is considered significant. An upstream regulator was considered increased (activated) if the $z$-score was $\geq 2$ (orange), and decreased (inhibited) if the $z$-score was $\leq-2$ (blue) 


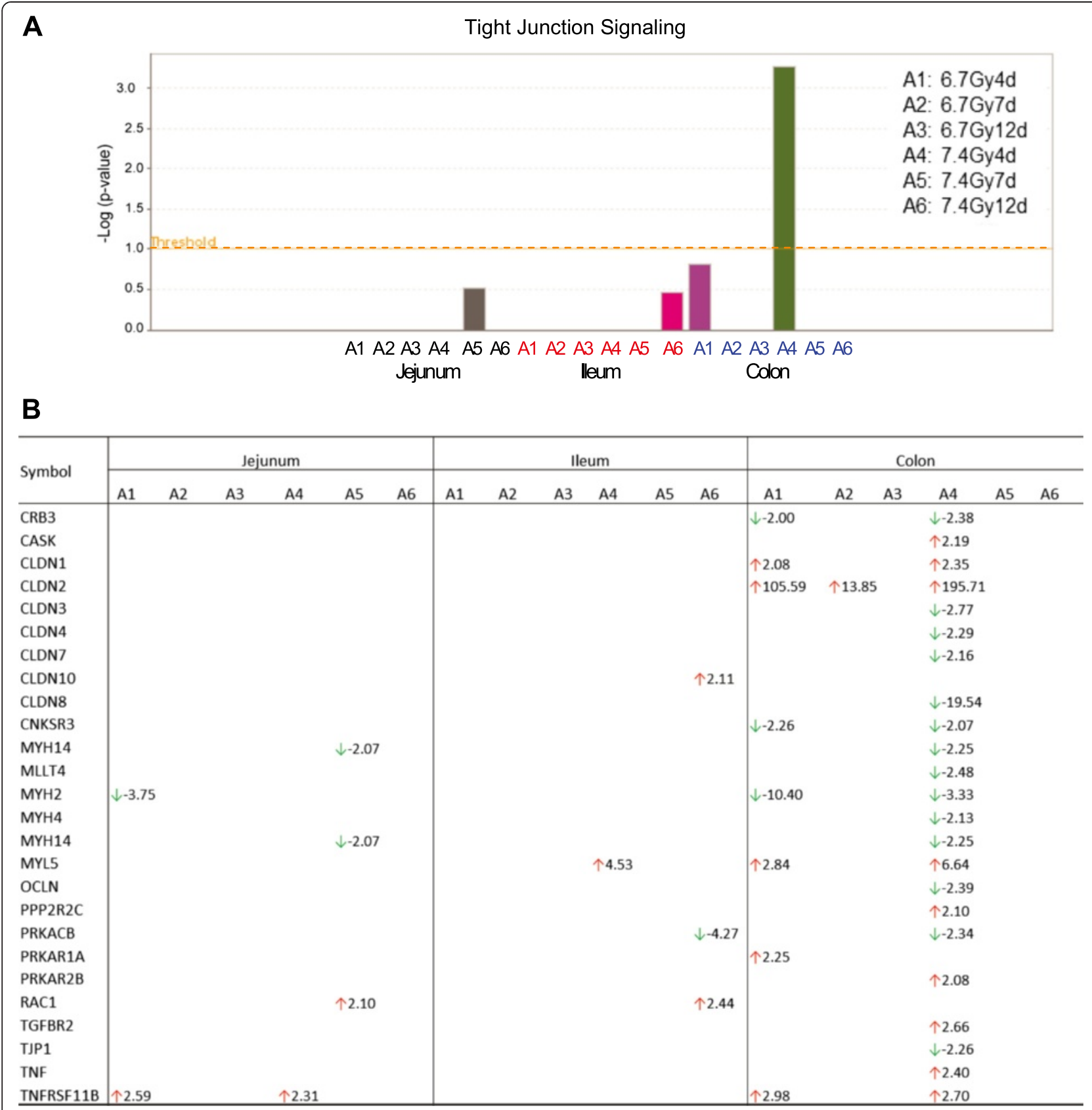

Fig. 4 Comparative analysis demonstrates that the tight junction signaling pathway is only activated in colon on day 4 after 7.4 Gy TBI (panel a). Pathways with a -log value ( $P$-value) above the threshold (dashed line) were significantly activated. Altered transcripts involved in tight junction signaling in jejunum, ileum and colon at the different time points after $6.7 \mathrm{~Gy}$ and $7.4 \mathrm{~Gy}$ TBI (panel b). The font color of A1 to A6 represents segment of jejunum (black), ileum (red) and colon (blue). A1 to A6 represent the time point of days 4, 7 and 12 after 6.7 Gy and 7.4 Gy TBI

of these matrix degradation genes inevitably causes cells to lose their attachment to the surrounding ECM. In addition, radiation induced strong expression of EDA2R, a member of the TNF receptor family that mediates p53-regulated anoikis [23]. Anoikis is a form of programmed cell death induced by detachment of anchorage-dependent cells from the surrounding ECM. EDA2R protein negatively regulates focal adhesion kinase, a central component of focal adhesion. In humans, activated EDA2R gives rise to a clinical syndrome characterized by loss of hair, sweat glands and teeth [24]. We hypothesize that activation of the EDA2R and TNF $\alpha$ cascades plays a key role in radiation-induced mucosal shedding in colon. Interestingly, these matrix degradation genes showed a much lower increase in jejunum and ileum than in colon. 


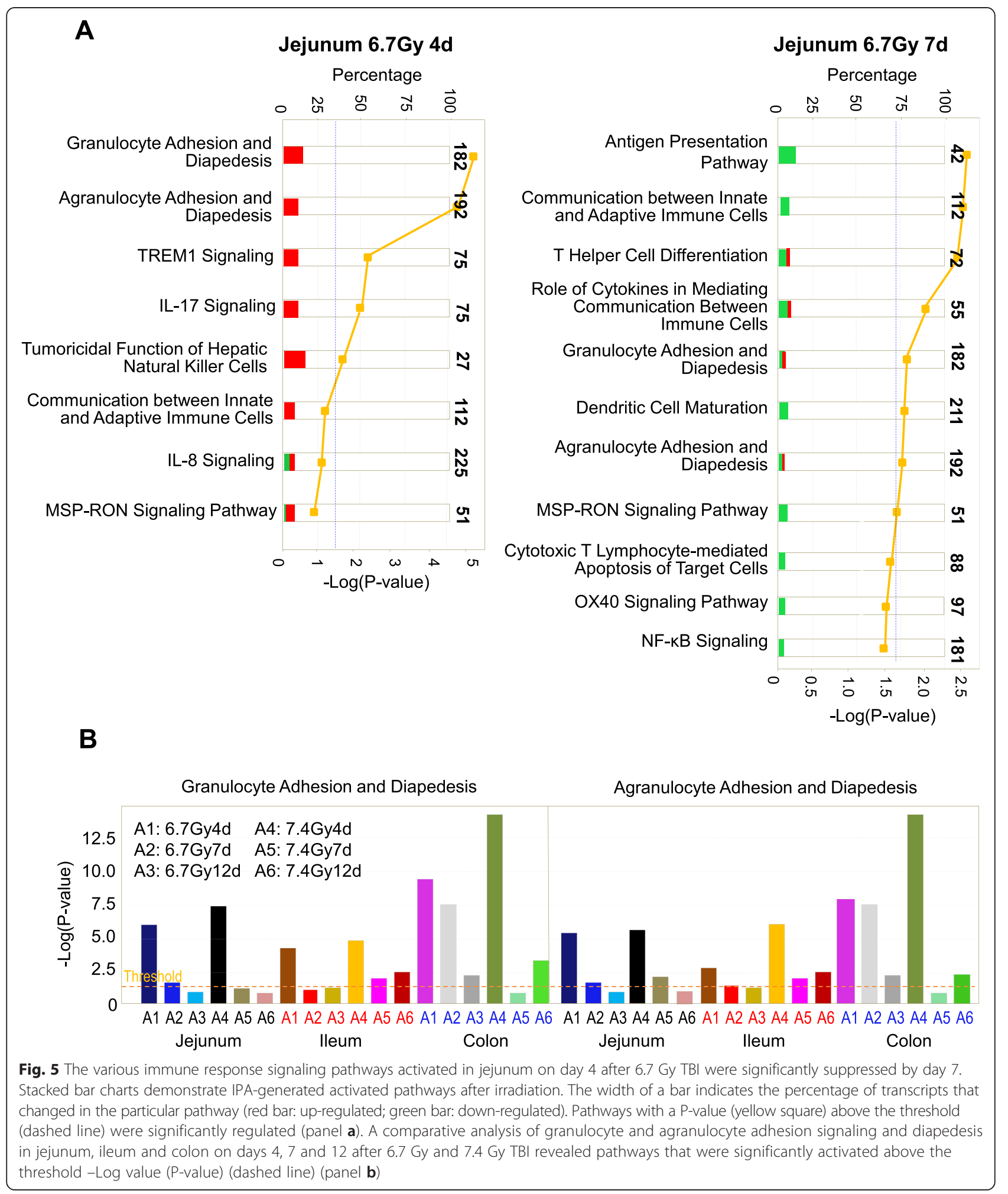

Intestinal mucosal barrier disruption in response to TBI changes the barrier's permeability. Our investigation of the expression of epithelial tight junction-associated genes in the different GI segments after irradiation revealed that only colon exposed to 7.4 Gy TBI caused significant ( $\geq 2$-fold) changes in numerous tight junction genes at day 4, with very few alterations in expression of tight junction-associated genes observed in jejunum and 


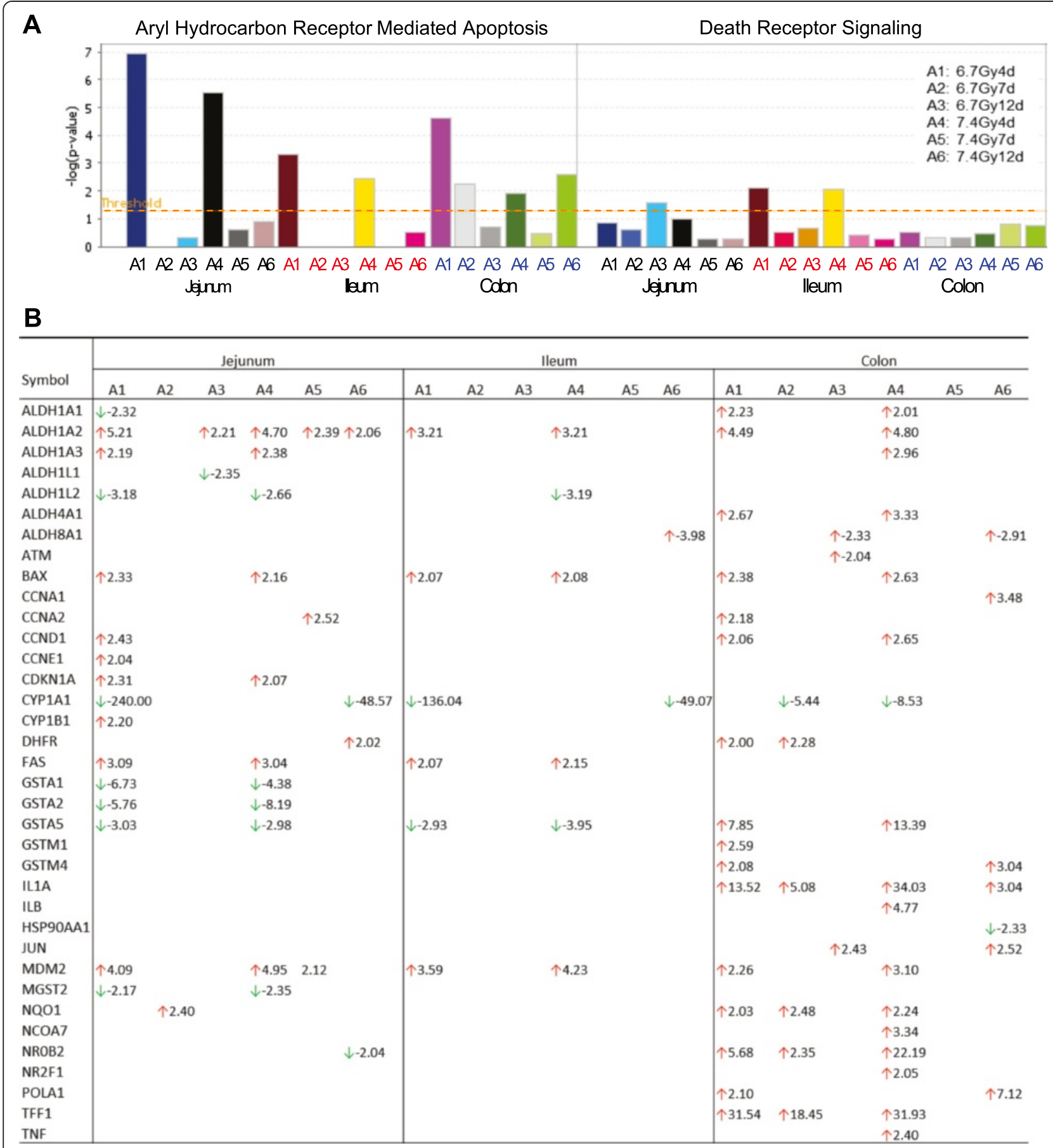

Fig. $6 \mathrm{TBI}$ induced significant activation of the AhR-mediated apoptosis signaling pathway in jejunum, ileum and colon at the early time point of 4 days after TBI. Pathways with a -log value (P-value) above the threshold (dashed line) were significantly activated (panel a). Genes involved in AhR signaling altered at the different time points after 6.7 Gy and 7.4 Gy TBI (panel b)

ileum (see Fig. 4a). In intestine, tight junction proteins form a barrier against unlimited paracellular passage of solutes and water; they also form channels allowing distinct permeation across the barrier $[25,26]$. Among the tight junction-associated genes that were up-regulated after irradiation was CLDN2, which showed striking
106- and 196-fold increases after 6.7 Gy and 7.4 Gy TBI, respectively. CLDN2 protein (claudin 2) has been shown to be of great clinical impact. It is a predominant poreforming protein for selective transportation of water and small cations (e.g., $\mathrm{Na}+$ and $\mathrm{K}+$ ) [27-29]. An increase of CLDN2 protein expression causes excessive diffusion of 


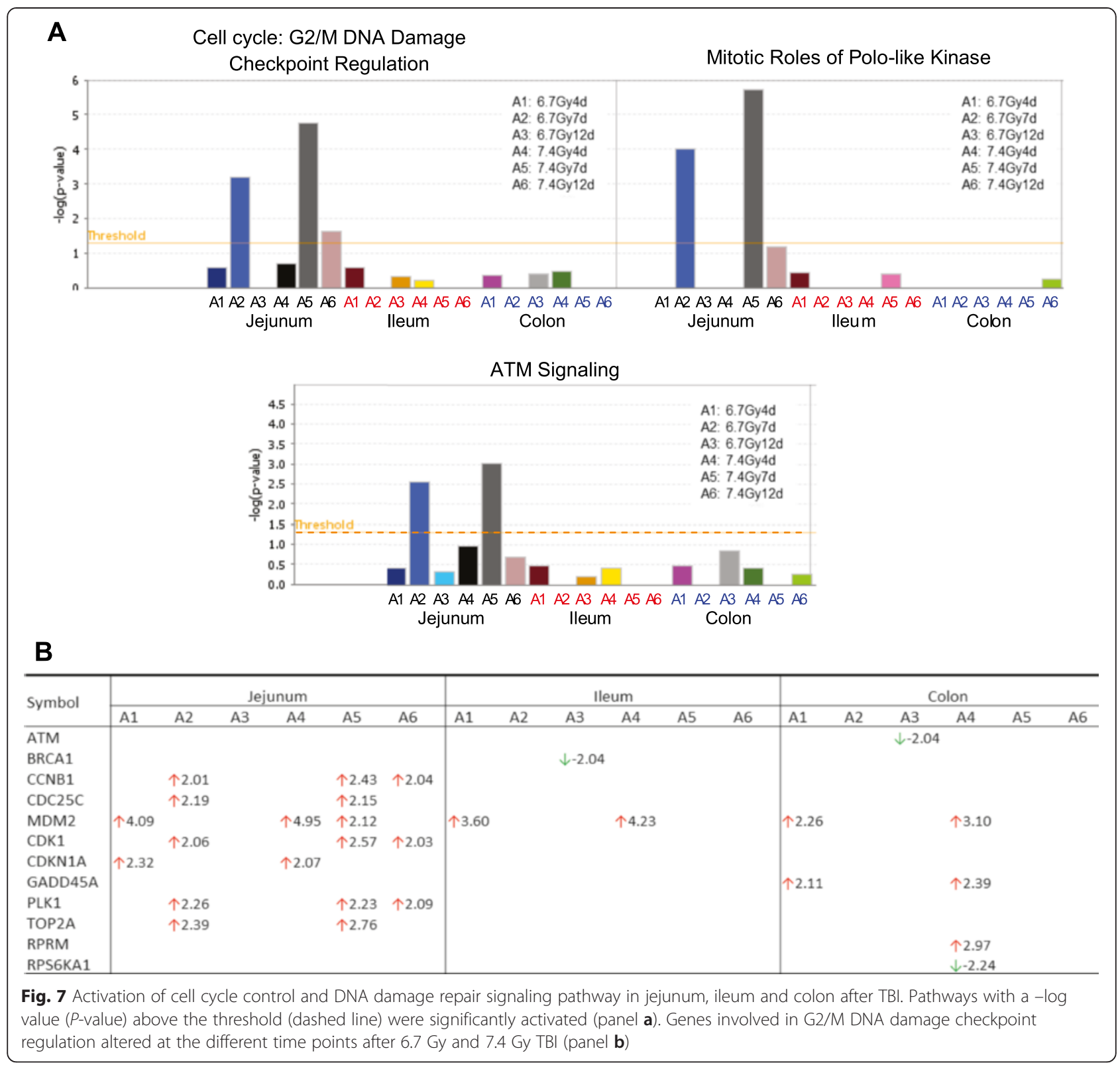

ions and water from blood to lumen, leading to leak-flux diarrhea $[27,28]$. CLDN2 expression can be induced by $\mathrm{TNF} \alpha$ and has been reported in various diseases, including Crohn's disease, ulcerative colitis and celiac disease $[27,28]$. In patients with Crohn's disease, the expression of CLDN2 is dramatically up-regulated, especially in patients with low expression of the sealing tight junction protein CLDN8 (claudin 8) [28]. In our studies, upregulated expression of CLDN2 after 7.4 Gy TBI was also accompanied with a wide down-regulation of sealing tight junction genes, including CLDN8, which was downregulated 20 -fold. These data suggest a direct link between tight junction disruption and the diarrhea observed in most of the irradiated animals from days 2 to 7 .
Activation of the TNF $\alpha$ cascade and tight junction signaling suggests that colon suffered the severest mucosal barrier disruption after TBI. This conclusion is further supported by the activation of immune response signaling pathways observed among the segments. Breakdown of the mucosal barrier causes bacterial translocation into the deep gut tissue and triggers an immune response and inflammation. Activation of these signaling pathways enables the organism to defend itself against infectious microbes. The magnitude of activation reflects the degree of tissue injury. Among the segments studied, colon had the greatest number of altered immune response transcripts and the highest magnitude of change. For example, the IL1A gene was 


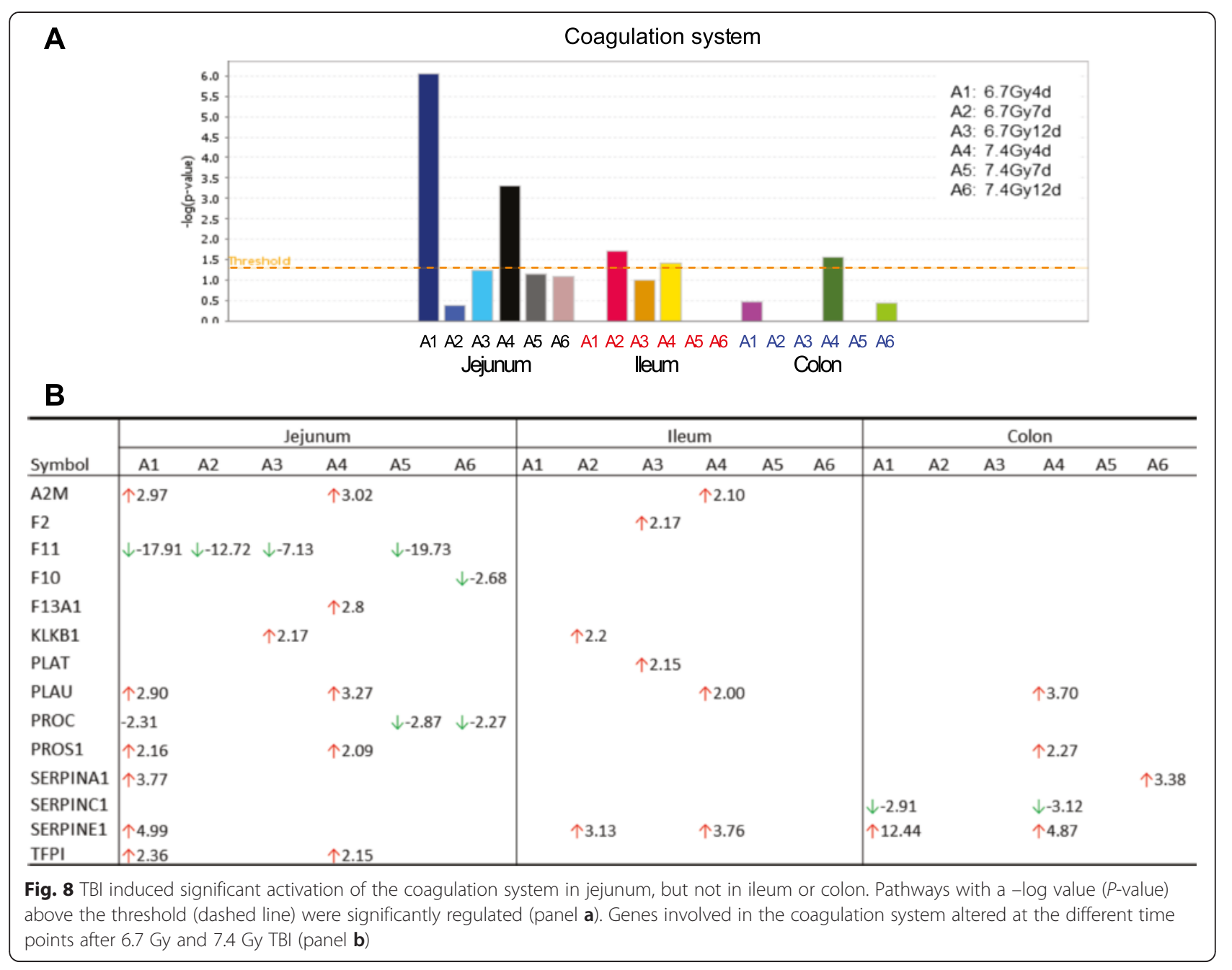

increased 14- and 34-fold in colon at day 4 after 6.7 Gy and 7.4 Gy TBI, respectively, but was not induced in jejunum and ileum. The same was the case with CCL11, CCL23, CXCL16, CXCL17, MMP9 and CLDN2. These transcripts were markedly up-regulated in colon but not induced in jejunum and ileum. In addition, CXCL8 was increased 61- and 56-fold in colon at day 4 after 6.7-Gy and 7.4-Gy TBI, respectively, but only 7- and 4fold in jejunum and ileum after the higher-dose TBI. These data seem reasonable in view of the disruption of the epithelial barrier in colon, the largest reservoir of microorganisms [30,31], which would prompt a strong defensive reaction to fight against the invading pathogens. As was the case with activation of the TNF $\alpha$ cascade and tight junction signaling, activation of the immune response was time-dependent, with day 4 as the most activated time point. At days 7 and 12, the activated immune response pathways were significantly suppressed.

In contrast to the colon, where TNF $\alpha$ cascade, tight junction and immune response signaling were most highly activated; jejunum was the segment with the strongest activation of apoptosis, cell cycle control/ DNA damage repair and coagulation system signaling after irradiation. After radiation exposure, a wave of apoptosis normally occurs within a few hours. But in this study, we found significant activation of AhRmediated apoptotic signaling at day 4 after both doses of TBI, with jejunum the most activated segment. AhR was originally characterized as a regulator of xenobiotic metabolism and currently is considered an important mediator of toxic responses [32, 33]. Activation of AhR signaling could be associated with the second wave of apoptosis induced by the toxicity generated by reactive oxygen species, ruptured cells or invasive microorganisms. TBI induced a wide activation of pathways that regulate cell cycle control and DNA damage repair [34, 35]. Surprisingly, activation of various DNA damage repair pathways occurred in jejunum, not in ileum or colon. The lack of DNA damage repair might explain why colon has a higher incidence of adenocarcinoma [36]. Previous studies, 


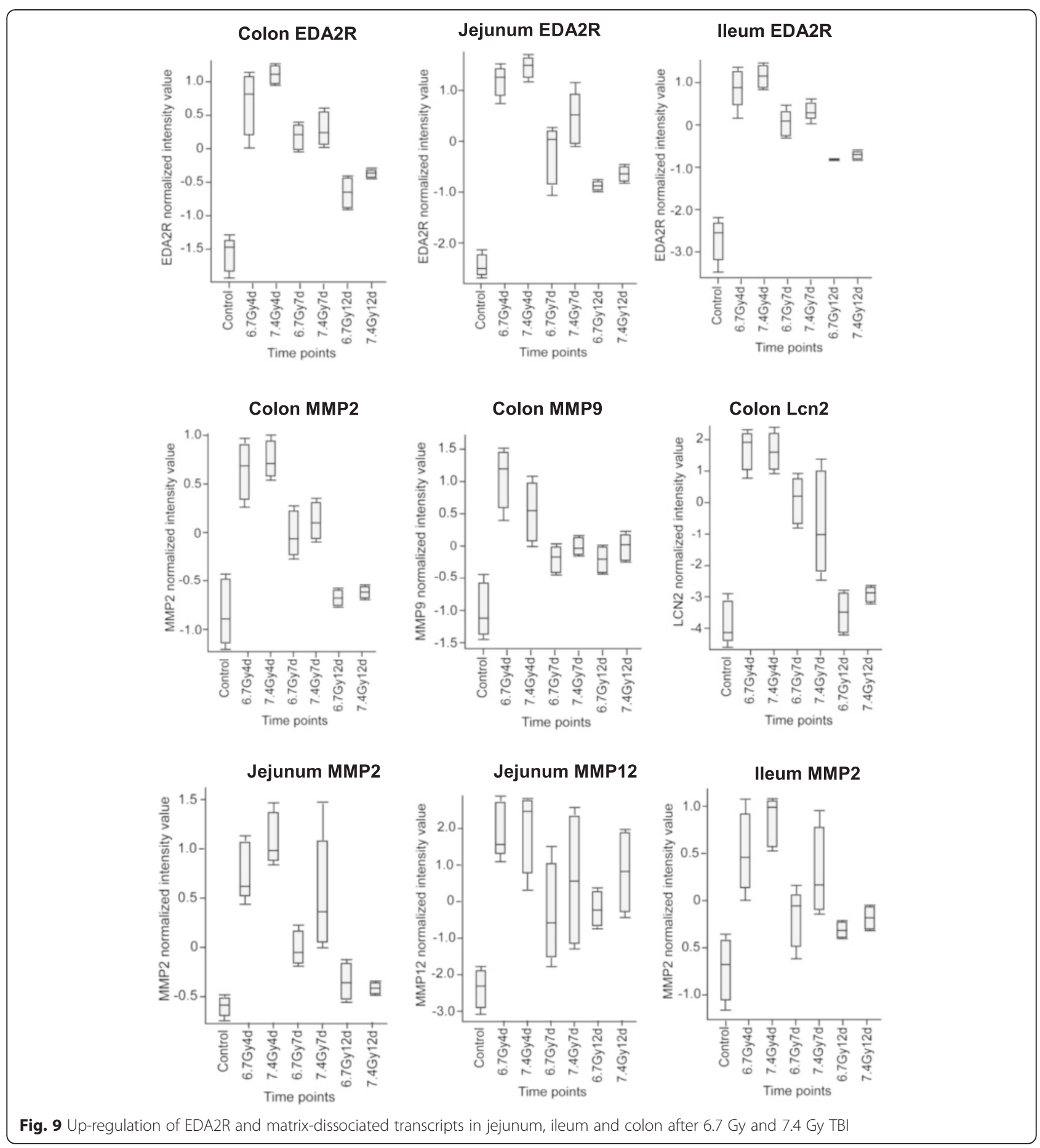

including ultrastructural studies, have shown that endothelial cells are also among the most radiationsensitive cell types $[37,38]$. Radiation induced a significant activation of coagulation system signaling in jejunum at day 4 . The strongest activation of the coagulation system in jejunum, but not ileum or colon, could be due to the abundance of blood capillaries in jejunal villi for nutrient absorption [39, 40].

\section{Conclusions}

This is the first systematic comparative study of the molecular and cellular responses to radiation injury in jejunum, ileum and colon. Activation of signaling cascades suggests that colon undergoes the most severe mucosal barrier interruption and inflammation and that jejunum experiences the most DNA damage and repair, apoptosis and endothelial dysfunction. These more pronounced 


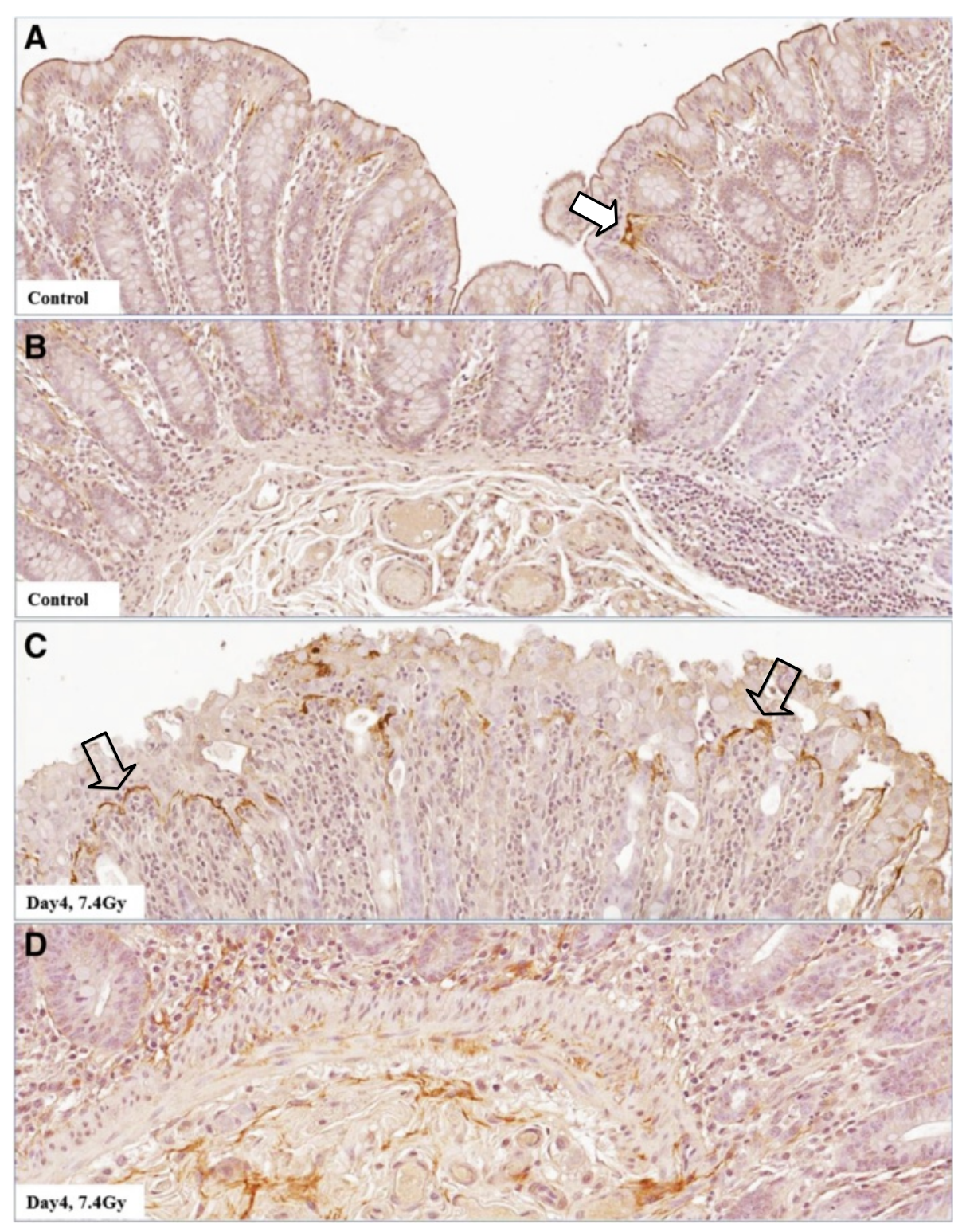

Fig. 10 Immunohistochemistry in colon tissue sections for TNFa protein. In unirradiated colon, TNFa protein is weakly distributed across the section; however, a streak of some visible signals can be seen beneath the intact mucosal epithelial lining (panels $\mathbf{a}$ and $\mathbf{b}$, solid white arrow). At day 4 after 7.4-Gy TBI, TNFa staining in the interstitial space from serosa to mucosa and submucosa of the colon exhibits significantly increased TNFa staining. Note the strong streak of TNFa staining in the mucosal region close to the epithelial barrier, forming an interface between swollen/ disrupted cells and normal-shaped cells (panels $\mathbf{c}$ and $\mathbf{d}$, open arrows)

alterations correlate with the high incidence of macroscopic pathologies that are observed in the colon after total body irradiation. Compared to jejunum and colon, ileum is the most resistant intestinal segment to radiation injury. The strongest activation of TNF $\alpha$ cascades and the striking up-regulation of its downstream matrix-dissociated genes suggest that TNF $\alpha$ modulation could be a target for mitigating radiationinduced mucosal barrier disruption. Future studies will verify these findings at the post-transcriptional and post-translational levels.

\section{Methods}

Animal model, intestinal tissue collection and total RNA preparation

Procedures involving the care and use of animals in this study were reviewed and approved by the Institutional Animal Care and Use Committee (IACUC) prior to conducting the experiments. All procedures were conducted according to the Guide for the Care and Use of Laboratory Animals (8th Ed., National Academy of Sciences) in the Association for Assessment and Accreditation of Laboratory Animal Care (AAALAC) accredited facility. 
Veterinary cares were available $24 \mathrm{~h} / 7 \mathrm{~d}$ throughout the study. The animal room environment was controlled (temperature $21 \pm 3{ }^{\circ} \mathrm{C}$, humidity $30-70 \%, 12 \mathrm{~h}$ light, $12 \mathrm{~h}$ dark, $10-15$ air changes per h) and temperature and relative humidity were monitored continuously. A standard certified commercial primate chow (Certified Primate Diet 2055CTM, Harlan Teklad, Madison, WI, USA) was available to each animal twice daily. Enrichment included fruits and other treats, television and radio.

At total of 24 young-adult male Rhesus macaque primates without known preexisting disease underwent sham-irradiation or exposure to a single uniform totalbody dose of 6.7-Gy (LD70/30) or 7.4-Gy (LD90/30) of gamma radiation from a cobalt-60 source (Theratron 1000, Best Theratronics, Ottawa, Ontario, Canada). The duration of the observation period before euthanasia was 4,7 and 12 days following TBI. Intestinal tissues from jejunum, ileum and colon were collected from unirradiated control $(n=4)$ and irradiated animals on days $4(n=4), 7(n=4)$, and $12(n=2)$ after 6.7-Gy and 7.4Gy TBI and stored in RNAlater. The same unirradiated controls $(n=4)$ were used for both 6.7-Gy and 7.4-Gy exposures. The length of each segment is around $3 \mathrm{~cm}$ and whole gut tissue $(\sim 50 \mathrm{mg})$ was used for tRNA extraction.

Total RNA was extracted with the use of RNeasy microarray tissue mini kits (Qiagen, Germantown, MD) and cleaned by TURBO DNase (Life Technologies/ Thermo Fisher Scientific, Grand Island, NY). The purity of RNA (OD260/280, OD260/230) was measured by NanoDrop 2000c spectrophotometer (Thermo Fisher Scientific, Waltham MA). RNA integrity was evaluated on a Bioanalyzer 2100 (Agilent Technologies, Santa Clara, CA). RNA integrity number (RIN) values below 6 were considered failed and not sent for microarray processing.

\section{Verification of gene expression by RT-qPCR}

Gene expression was verified with the same sets of total RNA samples sent for microarray. For each RNA sample, $2 \mu \mathrm{g}$ was used as the template for cDNA synthesis by using the High-Capacity cDNA reverse transcription kit (Life Technologies/Thermo Fisher Scientific). Steadystate mRNA levels were measured with real-time quantitative RT-qPCR using the following predesigned TaqMan (Life Technologies/Thermo Fisher Scientific) gene expression assays for the following genes: LCN15, ITM2A, EDA2R, MMP12, HBB, HBA2 and GALC. PCR amplification and detection were carried out on an ABI Prism 7000 sequence detection system (Life Technologies/ Thermo Fisher Scientific). Transcript levels were normalized to GAPDH and calculated relative to unirradiated controls by the standard $\Delta \Delta \mathrm{Ct}$ method. A statistics analysis was performed to estimate the consistency between RT-qPCR results and the microarray data.

\section{Gene microarray and data analysis}

A total 72 RNA samples (Table 3) extracted from jejunum, ileum and colon from un-irradiated control $(n=$ $4)$ and irradiated animals on days $4(n=4), 7(n=4)$, and $12(n=2)$ after $6.7-$ Gy and 7.4-Gy TBI were processed for gene microarray analysis without pooling. For each sample, $150 \mathrm{ng}$ of total RNA was converted to cDNA, amplified and labelled using the Low input quick amp one-color labelling kits from Agilent following the manufactures protocol. A total of $1.65 \mu \mathrm{g}$ of labelled cDNA was hybridized to Agilent single-color Rhesus Macaque (V2) gene expression 4 x $44 \mathrm{~K}$ slides (Agilent Technologies,). Arrays were washed, and data were analysed with Agilent's GeneSpring GX 11.0 software. Briefly, raw data were $\log 2$-transformed and then normalized to the 75th percentile of all values on a chip. Samples from unirradiated jejunum, ileum and colon were used as the baseline controls.

\section{Pathway analysis}

All transcripts that were differentially expressed more than 2-fold at $\mathrm{P}<0.05$ between TBI and control at each time point (Additional file 1: Table S1) were imported into Ingenuity Pathway Analysis (IPA) software (Qiagen/ Ingenuity, Redwood City, CA) for pathway analysis. Upstream Regulator Analysis in IPA was performed to identify the activation of upstream transcriptional regulators that could explain the observed gene expression changes. IPA Canonical Pathways Analysis was performed to identify the activation of pathways from a library of canonical pathways.

\section{Immunohistochemistry for TNFa}

Immunohistochemical staining was performed on colon tissue sections from control and irradiated animals with avidin-biotin complex $(\mathrm{ABC})$ technique, diaminobenzidine (DAB) chromogen, and hematoxylin counterstaining. Appropriate positive and negative controls were included. The primary antibody was rabbit polyclonal antibody to TNFa (1:100) (Abcam, Cambridge, MA). The dilution of the second antibody (goat anti-rabbit) was 1:400.

Table 3 Summary of 72 tRNA samples processed for microarray without pooling

\begin{tabular}{lllll}
\hline Segments dose & Control & 4 days & 7 days & 12 days \\
\hline Jejunum 6.7Gy & $N=4$ & $N=4$ & $N=4$ & $N=2$ \\
Jejunum 7.4Gy & & $N=4$ & $N=4$ & $N=2$ \\
lleum 6.7Gy & $N=4$ & $N=4$ & $N=4$ & $N=2$ \\
lleum 7.4Gy & & $N=4$ & $N=4$ & $N=2$ \\
Colon 6.7Gy & $N=4$ & $N=4$ & $N=4$ & $N=2$ \\
Colon 7.4Gy & & $N=4$ & $N=4$ & $N=2$ \\
\hline
\end{tabular}




\section{Statistical analysis GeneSpring analysis}

The expression profiles of unirradiated control samples from jejunum, ileum and colon were used as the baseline for generating the list of altered genes at each time point after TBI. Lists of gene were generated by $t$-test and Benjamini-Hochberg multiple testing correction with a false-discovery rate $<0.05$. Significantly changed genes were selected with a cut-off of $\mathrm{P}<0.05$ and fold change $\geq 2.0$.

\section{IPA analysis}

The significance of the association between the data set and the function/canonical pathway was determined by the P-value, which was calculated by Fisher's exact test and Benjamini-Hochberg multiple testing correction. A P-value $<0.05$ indicated a statistically significant, nonrandom association. Activation Z-score was used to identify the biological function/pathway expected to be increased or decreased. Z-score was also used to identify transcription factors that were significantly activated or inhibited. An absolute z-score of $\geq 2$ was considered significant. Increased (activated) biological functions/pathways were identified by a $\mathrm{z}$-score $\geq 2$, and decreased (inhibited) functions/pathways by a $\mathrm{z}$-score $<-2$.

\section{Availability of supporting data}

The raw and normalized microarray data is available in Gene Expression Omnibus (GEO) with an accession number GSE74110 (https://urldefense.proofpoint.com/v2/url? $\mathrm{u}=\mathrm{http}-3 \mathrm{~A} \_$_www.ncbi.nlm.nih.gov_geo_query_acc.cgi-3Fa cc-3DGSE74110\&d=BQIEAg\&c=6vgNTiRn9_pqCD9hKx9 JgXN1VapJQ8JVoF8oWH1AgfQ\&r=W8LxzO8ZOKsC96K r1NIe4x1i6OCs2ec2O9G-iXxNmcI\&m=MlqtHsTLnRF0Q 6kFosq7gSr_vwvAm7tRBtRJghcCTGE\&s=xphwihznMe0A mr6MuXesIsMdlrBputIhKWDEjR5LrCg\&e=).

\section{Additional files}

Additional file 1: Table S1. An excel file containing 18 lists of genes altered $\geq 2.0$-Fold from jejunum, ileum and colon at days 4, 7 and 12 after 6.7-Gy and 7.4-Gy TBI including fold-change and P-values. (XLS 3319 kb)

Additional file 2: Table S2. Altered genes regulating granulocyte adhesion and diapedesis in jejunum, ileum and colon at days 4, 7 and 12 after $6.7 \mathrm{~Gy}$ and $7.4 \mathrm{~Gy}$ TBI. (PDF $127 \mathrm{~kb}$ )

\section{Abbreviations}

Gl: Gastrointestinal; TBI: Total body irradiation; TNFa: Tumor necrosis factor a; CLDN2: claudin 2; IPA: Ingenuity Pathway Analysis; AhR: Activation of aryl hydrocarbon receptor; ECM: Extracellular matrix; $A B C$ : Avidin-biotin complex; DAB: Diaminobenzidine; IACUC: Institutional Animal Care and Use Committee; AAALAC: Association for Assessment and Accreditation of Laboratory Animal Care; BARDA: Biomedical Advanced Research and Development Authority; ASPR: Assistant Secretary for Preparedness and Response.
Competing interests

The authors declare that they have no competing interests.

\section{Authors' contributions}

JZ carried out the experiments and contributed to the planning, data analysis, results interpretation and writing. JW contributed to the planning and sample processing. MP contributed to the animal model creation and sample collection. SA contributed to the animal model creation and sample collection. DZ contributed to the planning and results interpretation. DSL contributed to microarray processing and results interpretation. $\mathrm{MHJ}$ oversaw all aspect of the study and contributed to the planning, results interpretation and writing. All authors read and approved the final manuscript.

\section{Acknowledgement}

Here we thank the Winthrop P. Rockefeller Cancer Institute Genomics Core Laboratory at University of Arkansas for Medical Sciences for providing the RNA sample evaluation analysis. We also thank William J. Gabello for his great effort on the paper's revision and editing. The project has been partially funded with Federal funds from the Office of the Assistant Secretary for Preparedness and Response (ASPR), Biomedical Advanced Research and Development Authority (BARDA) [under contract \#: HHSO100201100045C]; by the National Institutes of Health (P20 GM109005), and by the US Veterans Administration (www.va.gov). The ideas presented are the authors' and not those of the US Government.

\section{Author details}

${ }^{1}$ Division of Radiation Health, University of Arkansas for Medical Sciences, Little Rock, Arkansas 72205, USA. ${ }^{2}$ CiToxLAB North America, Laval, Quebec, Canada, H7V 4B3. ${ }^{3}$ Integrative Biology and Pharmacology, University of Texas Medical School at Houston, Houston, TX 77030, USA. ${ }^{4}$ Surgical Service, Central Arkansas Veterans Healthcare System, Little Rock, Arkansas 72205, USA.

Received: 14 May 2015 Accepted: 29 October 2015

Published online: 21 November 2015

References

1. Hauer-Jensen M, Wang J, Boerma M, Fu Q, Denham JW. Radiation damage to the gastrointestinal tract: mechanisms, diagnosis, and management. Curr Opin Support Palliat Care. 2007;1:23-9. Review.

2. Keefe DM, Gibson RJ, Hauer-Jensen M. Gastrointestinal mucositis. Semin Oncol Nurs. 2004;20:38-47. Review.

3. Sansonetti PJ. War and peace at mucosal surfaces. Nat Rev Immunol. 2004;4:953-64

4. Hauer-Jensen M, Denham JW, Andreyev HJ. Radiation enteropathypathogenesis, treatment and prevention. Nat Rev Gastroenterol Hepatol. 2014:11:470-79. Review.

5. Hauer-Jensen M. Late radiation injury of the small intestine. Clinical, pathophysiologic and radiobiologic aspects. Acta Oncol. 1990;29:401-15. A review.

6. Potten CS, Merritt A, Hickman J, Hall P, Faranda A. Characterization of radiation-induced apoptosis in the small intestine and its biological implications. Int J Radiat Biol. 1994;65:71-8.

7. Hovdenak N, Wang J, Sung CC, Kelly T, Fajardo LF, Hauer-Jensen M. Clinical significance of increased gelatinolytic activity in the rectal mucosa during external beam radiation therapy of prostate cancer. Int J Radiat Oncol Biol Phys. 2002;53:919-27.

8. François A, Aigueperse J, Gourmelon P, Macnaughton WK, Griffiths NM. Exposure to ionizing radiation modifies neurally-evoked electrolyte transport and some inflammatory responses in rat colon in vitro. Int J Radiat Biol. 1998;73:93-101.

9. Leppkes M, Roulis M, Neurath MF, Kollias G, Becker C. Pleiotropic functions of TNFa in the regulation of the intestinal epithelial response to inflammation. Int Immunol. 2014;26:509-15.

10. Günther C, Martini E, Wittkopf N, Amann K, Weigmann B, Neumann H, et al. Caspase-8 regulates TNF-a-induced epithelial necroptosis and terminal ileitis. Nature. 2011:477:335-9.

11. Arnold JW, Klimpel GR, Niesel DW. Tumor necrosis factor (TNF alpha) regulates intestinal mucus production during salmonellosis. Cell Immunol. 1993;151:336-44 
12. Wang F, Graham WV, Wang Y, Witkowski ED, Schwarz BT, Turner JR. Interferon-gamma and tumor necrosis factor-alpha synergize to induce intestinal epithelial barrier dysfunction by up-regulating myosin light chain kinase expression. Am J Pathol. 2005;166:409-19.

13. Su L, Nalle SC, Shen L, Turner ES, Singh G, Breskin LA, et al. TNFR2 activates MLCK-dependent tight junction dysregulation to cause apoptosis-mediated barrier loss and experimental colitis. Gastroenterology. 2013;145:407-15.

14. Cotter TG. Apoptosis and cancer: the genesis of a research field. Nat Rev Cancer. 2009;9:501-7. Review.

15. Saelens X, Festjens N, Vande Walle L, van Gurp M, van Loo G, Vandenabeele $P$. Toxic proteins released from mitochondria in cell death. Oncogene. 2004;23:2861-74.

16. Packey $C D$, Ciorba MA. Microbial influences on the small intestinal response to radiation injury. Curr Opin Gastroenterol. 2010;26:88-94.

17. Biju PG, Garg S, Wang W, Choudhry MA, Kovacs EJ, Fink LM, et al. Procalcitonin as a predictive biomarker for total body irradiation-induced bacterial load and lethality in mice. Shock. 2012;38:170-6.

18. Thiagarajah JR, Gourmelon P, Griffiths NM, Lebrun F, Naftalin RJ, Pedley KC. Radiation induced cytochrome $\mathrm{c}$ release causes loss of rat colonic fluid absorption by damage to crypts and pericryptal myofibroblasts. Gut. 2000;47:675-84.

19. Watson AJ, Hughes KR. TNF-a-induced intestinal epithelial cell shedding: implications for intestinal barrier function. Ann NY Acad Sci. 2012;1258:1-8. Review.

20. Present DH, Rutgeerts $P$, Targan S, Hanauer SB, Mayer L, van Hogezand RA, et al. Infliximab for the treatment of fistulas in patients with Crohn's disease. N Engl J Med. 1999;340:1398-405.

21. Vandenbroucke RE, Vanlaere I, Van Hauwermeiren F, Van Wonterghem E, Wilson C, Libert C. Pro-inflammatory effects of matrix metalloproteinase 7 in acute inflammation. Mucosal Immunol. 2014;7:579-88.

22. Yan L, Borregaard N, Kjeldsen L, Moses MA. The high molecular weight urinary matrix metalloproteinase (MMP) activity is a complex of gelatinase B/MMP-9 and neutrophil gelatinase-associated lipocalin (NGAL). Modulation of MMP-9 activity by NGAL. J Biol Chem. 2001;276:37258-65.

23. Tanikawa C, Furukawa Y, Yoshida N, Arakawa H, Nakamura Y, Matsuda K. XEDAR as a putative colorectal tumor suppressor that mediates p53regulated anoikis pathway. Oncogene. 2009;28:3081-92.

24. Brosh R, Sarig R, Natan EB, Molchadsky A, Madar S, Bornstein C, et al. p53dependent transcriptional regulation of EDA2R and its involvement in chemotherapy-induced hair loss. FEBS Lett. 2010;584:2473-77.

25. Ménard S, Cerf-Bensussan N, Heyman M. Multiple facets of intestinal permeability and epithelial handling of dietary antigens. Mucosal Immunol. 2010;3:247-59.

26. Schmitz H, Barmeyer C, Fromm M, Runkel N, Foss HD, Bentzel CJ, et al. Altered tight junction structure contributes to the impaired epithelial barrier function in ulcerative colitis. Gastroenterology. 1999;116:301-9.

27. Ahmad R, Chaturvedi R, Olivares-Villagómez D, Habib T, Asim M, Shivesh P, et al. Targeted colonic claudin-2 expression renders resistance to epithelial injury, induces immune suppression, and protects from colitis. Mucosal Immunol. 2014;7:1340-53.

28. Zeissig S, Bürgel N, Günzel D, Richter J, Mankertz J, Wahnschaffe U, et al. Changes in expression and distribution of claudin 2, 5 and 8 lead to discontinuous tight junctions and barrier dysfunction in active Crohn's disease. Gut. 2007:56:61-72.

29. Schulzke JD, Ploeger S, Amasheh M, Fromm A, Zeissig S, Troeger $\mathrm{H}$, et al. Epithelial tight junctions in intestinal inflammation. Ann N Y Acad Sci. 2009;1165:294-300.

30. Stephen AM, Cummings JH. The microbial contribution to human faecal mass. J Med Microbiol. 1980;13:45-56.

31. Savage DC. Microbial ecology of the gastrointestinal tract. Annu Rev Microbiol. 1977;31:107-33.

32. Marlowe JL, Puga A. Aryl hydrocarbon receptor, cell cycle regulation, toxicity, and tumorigenesis. J Cell Biochem. 2005;96:1174-84. Review.

33. Kamath $A B, X u H$, Nagarkatti PS, Nagarkatti M. Evidence for the induction of apoptosis in thymocytes by 2,3,7,8-tetrachlorodibenzo-p-dioxin in vivo. Toxicol Appl Pharmacol. 1997;142:367-77.

34. Zheng J, Garg S, Wang J, Loose DS, Hauer-Jensen M. Laser capture microdissected mucosa versus whole tissue specimens for assessment of radiation-induced dynamic molecular and pathway changes in the small intestine. PLoS One. 2013;8:e53711.
35. Lund PK. Fixing the breaks in intestinal stem cells after radiation: a matter of DNA damage and death or DNA repair and regeneration. Gastroenterology. 2012;143:1144-7.

36. Peltomaki P. Role of DNA mismatch repair defects in the pathogenesis of human cancer. J Clin Oncol. 2003;21:1174-9.

37. Paris F, Fuks Z, Kang A, Capodieci P, Juan G, Ehleiter D, et al. Endothelial apoptosis as the primary lesion initiating intestinal radiation damage in mice. Science. 2001;293:293-7.

38. Wang J, Boerma M, Fu Q, Hauer-Jensen M. Significance of endothelial dysfunction in the pathogenesis of early and delayed radiation enteropathy. World J Gastroenterol. 2007;13:3047-55.

39. Machovich R. Blood coagulation-fibrinolytic system and endothelial cells Acta Biochim Biophys Acad Sci Hung. 1985;20:135-53.

40. Stern D, Nawroth P, Handley D, Kisiel W. An endothelial cell- dependent pathway of coagulation. Proc Natl Acad Sci U S A. 1985;82:2523-7.

\section{Submit your next manuscript to BioMed Central and take full advantage of:}

- Convenient online submission

- Thorough peer review

- No space constraints or color figure charges

- Immediate publication on acceptance

- Inclusion in PubMed, CAS, Scopus and Google Scholar

- Research which is freely available for redistribution 